Vol. 4, Special Issue 2, January 2017

\title{
Zigbee Based Wireless A Remote Carbon Dioxide (CO2) Monitoring System
}

\author{
Priti P. Chavan ${ }^{1}$, Surpiya S. Kadam ${ }^{2}$ \\ Student, E\&TC Dept, Daulatrao Aher College of Engg, Karad, India ${ }^{1}$ \\ Assistant Professor, E\&TC Dept, Daulatrao Aher College of Engg, Karad, India ${ }^{2}$
}

\begin{abstract}
The increasing quantity of $\mathrm{CO} 2$ is a big problem leading to global warming. Therefore it is necessary to properly design a system to monitor the amount of $\mathrm{CO} 2$ in the atmosphere to prevent the debacles of future.This paper gives us details about how to develop CO2 monitoring system based on the ZigBee protocol. Graphical user interface is helpful to display monitoring data on screen. This is another important advantage present in this paper.
\end{abstract}

Keywords: CO2 Concentration, Controller, Monitoring, Real time, Zigbee.

\section{INTRODUCTION}

The object of the project is to measure concentrate/ion of carbon dioxide (Co2). Here we use the $\mathrm{Co} 2$ sensor to measure the gas in air. In today's world high speed wireless systems are in demand. Wired communication cannot meet the requirements of speed and cannot be available every time everywhere. It is found in many industries and laboratories that the percentage of $\mathrm{Co} 2$ is very dangerous and bad for workers. So we are design wireless $\mathrm{Co} 2$ monitoring system which measure the $\mathrm{Co} 2$ amount in working area.This project wireless sensor networks a remote Carbone Dioxide (Co2) Concentration Monitoring System using Zigbee is a reliable circuit that take over the task of measuring the concentration of $\mathrm{Co} 2$ accurately. Zigbee is used for the data transmitter as well as receiver which is controlled by the microcontroller. The real time data display on the monitor shows the Co2 amount to the observer. According to the predefined limit of $\mathrm{Co} 2$, we create safe and dangerous zone on display. If the amount of $\mathrm{Co} 2$ exceeds the safe zone and enters in danger zone then observer getting this information at a time one alarm is used in this system. Zigbee is a new Wireless Protocol characterized by less distance and low speed. It can be used in special situation for signal collection, processing and transmitting. ZigBee is a technology now being used for wireless sensor networks. A sensor network consists of sensing, computing and communications elements that allows the administrator to instrument, observe and react to events and phenomena in a specified environment. The maximum area cover by Zigbee is $40 \mathrm{~m}$. In line of site area increased up to $110 \mathrm{~m}$.

\section{PROPOSED SYSTEM}

The basic block diagram of wireless $\mathrm{Co} 2$ monitoring system is shown in the fig. 1. This block diagram consist of the following essential blocks. 1. Co2 Sensor

2. ATMEGA-328P MICRO-CONTROLLER

3. ZIGBEE

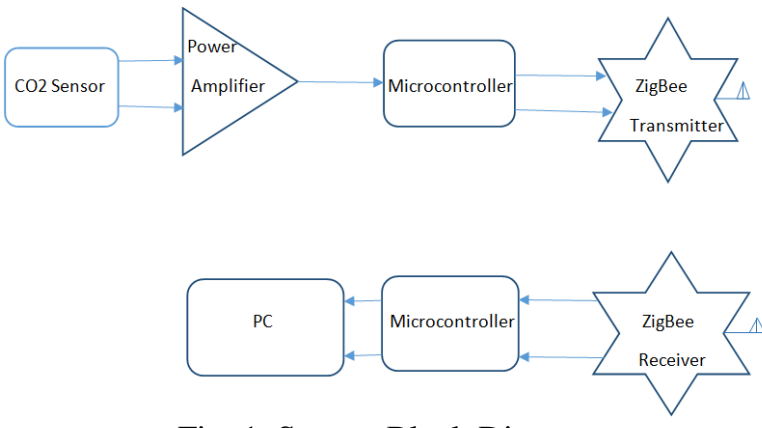

Fig. 1. System Block Diagram

In this project we are going to design a system which will wireless $\mathrm{Co} 2$ concentration monitoring. So firstly we have to measure the values of $\mathrm{Co} 2$ concentration by using the MQ135 sensor. After that by using ATMEGA-328p we are going to transmit these values wirelessly to control room via zigbee. At the control room we use a sensor unit basically consists of $\mathrm{Co} 2$ sensor used to detect the predetermined parameters that indicate the $\mathrm{Co} 2$ concentration. The sensor use battery for their operation. The information being sensed by the sensors is then converted into electrical signal and then it is passed to a microcontroller or microprocessor that processes it to the value understandable by humans. These values are then given to the ZigBee trans-receiver at the observer site.At the monitoring side, we slots the $\mathrm{Co} 2$ concentration levels according to the above figure.

\section{Arduino UNO:-}

Arduino is common term for a software company, project, user community designs and manufactures computer opensource hardware and software, and microcontroller-based kits for building digital devices and interactive objects that can sense and control physical devices.

\section{ZigBee:-}

ZigBee is an IEEE 802.15.4-basedspecification for a suite of high-level communication protocols used to create 
personal area networks with small, low-power digital radios. The technology defined by the ZigBee specification is intended to be simpler less expensive than other wireless personal area networks (WPANs), such as Bluetooth or Wi-Fi. Applications include wireless light switches, electrical meters in-home-displays, traffic management systems, and other costumer and industrial application that requires short-range low-rate wireless data transfer.

\section{ATMEGA-328P Micro-controller:-}

The Atmel ${ }^{\circledR}$ Pico Power ${ }^{\circledR}$ ATmega328/P is a low-power CMOS 8-bit microcontroller based on the $\mathrm{AVR}^{\circledR}$ enhanced RISC architecture. By executing powerful instructions in a single clock cycle, the ATmega328/P achieves throughputs close to $1 \mathrm{MIPS}$ per MHz. This empowers system designer to optimize the device for power consumption versus processing speed.

\section{MQ135 CO2 Sensor:-}

MQ-135 gas sensor applies $\mathrm{SnO} 2$ which has a lower conductivity in the clear air as a gas-sensing material. In an atmosphere where there may be polluting gas, the conductivity of the gas sensor raises along with the concentration of the polluting gas increases. MQ-135 performs a good detection to smoke and other harmful gas, especially sensitive to ammonia, sulfide and benzene steam. Its ability to detect various harmful gas and lower cost make MQ-135 an ideal choice of different applications of gas detection.

Features:-

- Wide detecting scope.

- Fast response and High sensitivity.

- Stable and long life.

- Have 6 pin, 4 of them are used to fetch signals, and other 2 are used for providing heating current.

- Range- $350 \sim 5000$ ppm.

\section{System Flow Chart:-}

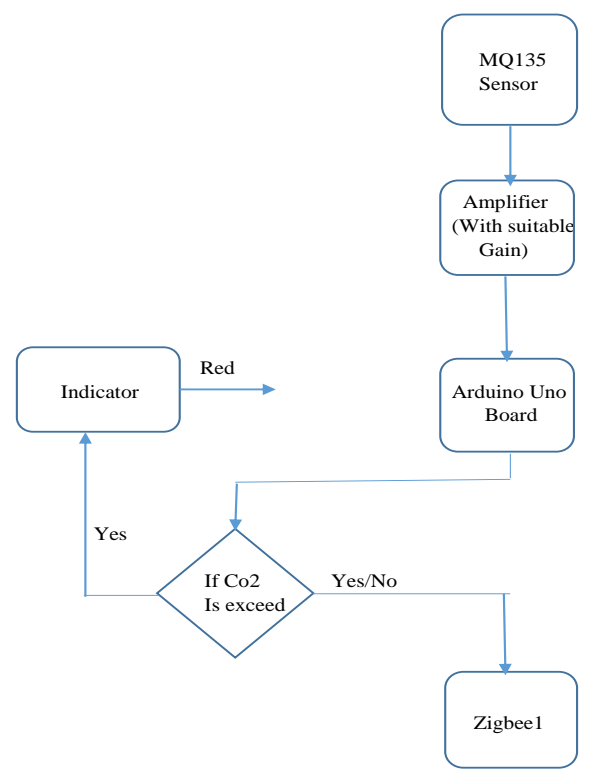

Fig.2. System Flow Chart
6. System Circuit Diagram:-

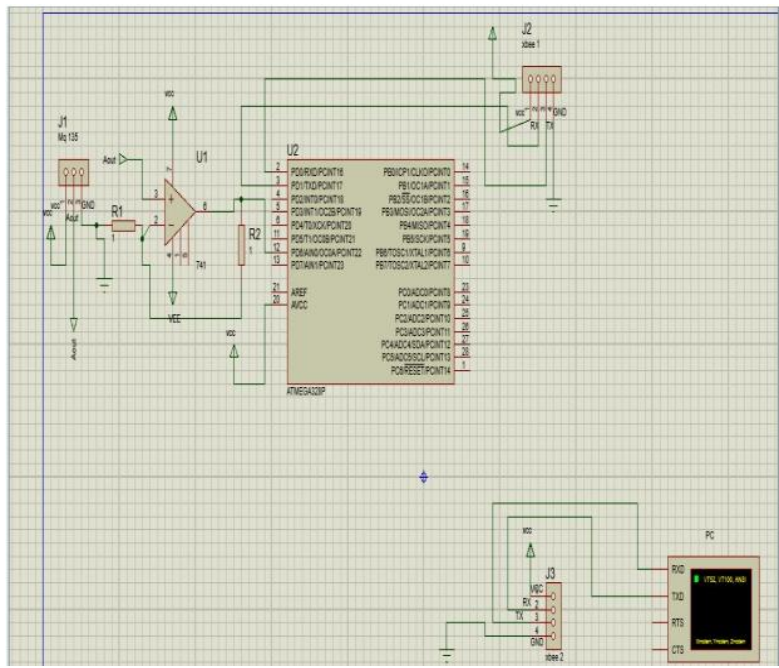

Fig.3.System Circuit Diagram

\section{REVIEW}

The increased concentration of greenhouse gas carbon dioxide increases the atmospheric temperature and causes the problems like global warming. Therefore, the solution for this problem is to capture and store the $\mathrm{CO} 2$ and use it for other purposes like oil and gas production. But these all efforts will be of no use if collected gas leaks from the storage reservoir. For this purpose the monitoring system with cost-effective sensors should be deployed to prevent leakage [1].

WSN is a modern information technology with the integration of sensor technology, automatic control technology, data transmission network, storage, processing and analysis technology. Compared with traditional monitoring techniques, WSN is featured by its low-cost, low power consumption, simple to deploy, without onsite maintenance, etc., and it can achieve a variety of regional low-cost unmanned continuous monitoring [2].

Carbon dioxide (CO2) concentration monitoring system developed, based on the technologies of wireless sensor networks, in allusion to the gas leakage monitoring requirement for $\mathrm{CO} 2$ capture and storage. The remote online $\mathrm{CO} 2$ monitoring system consists of monitoring equipment and a data centre server [3].

Experimentally it is proved that the hardware develop by Cypress Inc. is the best solution which works on low power with less complexity and high reliability for greenhouse control. In the future, if parameter still increase, then for WSN technology with currently available bandwidth, may not be sufficient. Then WSN with cognitive radio technology may be the solution [4].

WAPMS uses an Air Quality Index to categorize the various levels of air pollution. It also associates meaningful and very intuitive colors to the different categories, thus the state of air pollution can be communicated to the user very easily. The major motivation behind our study and the development of the 
system is to help the government to devise an indexing system to categorize air pollution in Mauritius. The system also uses the AQI to evaluate the level of health concern for a specific area [5].

A front end application was developed in .Net to acquire the transmitted air pollution data from the transmitter block. Here wireless communication is done through zigbee protocol. A zigbee transceiver module transmits the data from the arm processor (transmitter end) which is aquired from interfaced mq-7 mq-2 sensors. At the receiver end this. Net windows form application acquires the data from the receiver end zigbee transreciever module. This windows form application reads the data via selected com port (through which the zigbee module is connected) and finally the acquired data is made to store in the text file along with the date and time [6].

\section{IV.RESULT}

\section{Minimum CO2 Concentration Values:-}

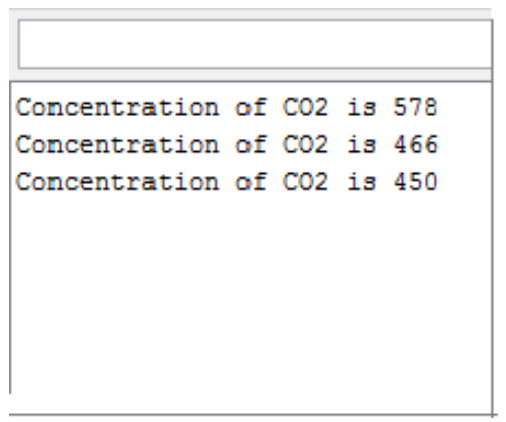

Fig.4. Real time data

\section{Maximum CO2 Concentration Values:-}

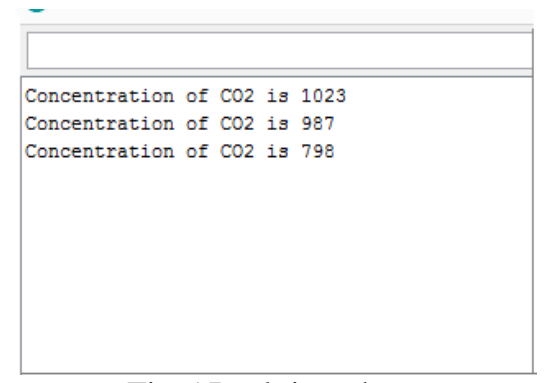

Fig.5.Real time data

\section{CONCLUSION}

By using this system we will be able to monitor the amount of $\mathrm{CO} 2$ in the working conditions. As today's demand of the workers motivation is proper working conditions. The quality of life of people will improve to a greater extent. According to the requirement of the industries, this system works at real time. This system measure theCO2 gas in working area to check whether gas cross the safety limit or not. When the amount of gas cross the safety limit then system indicates danger signal with real time and date.

\section{ACKNOWLEDGEMENT}

We express our sincere thanks to Ms. Kadam S.S. whose supervision, inspiration and valuable discussion has helped us tremendously to complete our project. His guidance proved to be the most valuable to overcome all the hurdles in the fulfillment of this Project. We grateful to Prof. Chorage P. J. for direct or indirect help in the completion of this project. Last but not least, this acknowledgement would be incomplete without rendering my sincere gratitude to all those who have helped us in the completion of this project.

\section{REFERENCES}

[1] Ms. Wagh Sushama Mohan, Prof. Devi R.J., "Monitoring of Geological CO2 based on Wireless Sensor Network", International Journal of Engineering Sciences \& Research Technology, December 2014.

[2] Raja Vara Prasad Y, Mirza Sami Baig, Rahul K Mishra, P. Rajalashmi, U.B. Desai and S.N. Merchant, "Real Time Wireless Air Pollution Monitoring System", ICTACT Journal on Communication Technology: Special Issue on Next Generation Wireless Network and Application, Volume-2, Issue-2, June 2011.

[3] K. Raghava Rao, Monica Vallabhaneri, Srikanth Narayanaraju, Rajendra Kumar Jonnalagadda, Sunilkumar Kanaparthi, "Air Pollution Monitoring Using Zigbee Based Wireless Sensor Networks", International Journal of Electronics and Communication Engineering \& Technology (IJECET), ISSN 0976 - 6464(Print), ISSN 0976 - 6472(Online), Volume 5, Issue 5, May .

[4] D.D. Chaudhary, S.P. Nayse, L.M. Waghmare, "Application of Wireless Sensor Network for Greenhouse Parameter Control in Precision Agriculture", International Journal of Wireless \& Mobile Networks (IJWMN) Vol. 3, No. 1, February 2011.

[5] R.A. Roseline, Dr. M. Devapriya, Dr. P. Sumathi, "Pollution Monitoring Using Sensor And Wireless Sensor Networks" International Journal of Application or Innovation in Engineering \& Management (IJAIEM), Volume 2, Issue 7, July 2013.

[6] Kondamudi Siva Sai Ram, A.N.P.S. Gupta, "IoT Based Data Logger System For Weather Monitoring Using Wireless Sensor Networks", International Journal of Engineering Trends and Technology (IJETT) - Volume 32 Number 2. 